\title{
Area and Length Minimizing Flows for Shape Segmentation
}

\author{
Kaleem Siddiqi \\ Yale University, Center for \\ Computational Vision \& Control \\ siddiqi-kaleem@cs.yale.edu \\ Allen Tannenbaum \\ University of Minnesota \\ Dept. of Electrical Engineering \\ tannenba@ee.umn.edu
}

\author{
Yves Bérubé Lauzière \\ McGill University \\ Cent. for Intelligent Machines \\ berube@cim.mcgill.ca \\ Steven W. Zucker \\ Yale University, Center for \\ Computational Vision \& Control \\ zucker-steven@cs.yale.edu
}

\begin{abstract}
Several active contour models have been proposed to unify the curve evolution framework with classical energy minimization techniques for segmentation, such as snakes. The essential idea is to evolve a curve (in 2D) or a surface (in 3D) under constraints from image forces so that it clings to features of interest in an intensity image. Recently the evolution equation has been derived from first principles as the gradient flow that minimizes a modified length functional, tailored to features such as edges. However, because the flow may be slow to converge in practice, a constant (hyperbolic) term is added to keep the curve/surface moving in the desired direction. In this paper, we provide a justification for this term based on the gradient flow derived from a weighted area functional, with image dependent weighting factor. When combined with the earlier modified length gradient flow we obtain a pde which offers a number of advantages, as illustrated by several examples of shape segmentation on medical images. In many cases the weighted area flow may be used on its own, with significant computational savings.
\end{abstract}

\section{Introduction}

In the application of curve evolution theory to visual shape analysis, Kimia, Tannenbaum and Zucker introduced a reaction-diffusion space for shape representation $[11,13,12]$. Using a reaction-diffusion model from mathematical physics, a planar shape is evolved with a velocity vector in the direction normal to the moving front, which consists of two terms: a constant (hyperbolic) term, and a curvature (parabolic) term. The key idea is to play off one term against the other: the constant motion term leads to the formation of shocks from which a representation of shape can be derived, and the diffusive curvature term smoothes the front, which is essential for distinguishing more significant shape features from less significant ones.

This type of technique was introduced into shape modelling by Casselles et al. [4] and Malladi et al. [14], via the addition of a multiplicative image gradient stopping term. This has led to powerful new techniques for edge capturing. These active contour models have a significant advantage over classical snakes, in that changes in topology due to the splitting and merging of multiple contours are handled in a natural way. Along these lines, Tek and Kimia [23] have further suggested a reaction-diffusion space of bubbles, where in place of a single contour, a number of bubbles are simultaneously placed and grown from homogeneous regions of the image.

Against this background, in several independent works $[5,9,10,20]$ a new active contour model was proposed which unified the curve evolution approaches with the classical energy minimization methods [8]. The technique is motivated by the Euclidean curve shortening equation (interpret the gradient direction as that in which the Euclidean perimeter is shrinking as fast as possible). The key is to multiply the Euclidean arc-length by a function tailored to the features of interest in the intensity image, and then to write down the resulting gradient evolution equations. Mathematically, this amounts to defining a new metric in the plane tailored to the given image, and then computing the corresponding gradient flow. This leads to new snake models which efficiently attract the evolving front to features such as edges. A viscosity analysis demonstrates the existence and uniqueness of a solution to the partial differential equation and provides 
theoretical justification for its use.

Whereas the above method motivates the use of a curvature term in shape modelling, such flows can be unacceptably slow to converge in practice. Therefore, the authors of $[5,9,10,20]$ follow $[4,14]$ by adding a constant inflation term to keep the curve moving in the desired direction. Our main contribution in this paper is the replacement of this hyperbolic term with one derived from minimizing a certain weighted area energy functional. More precisely, in analogy to the case of Euclidean arc-length we multiply the infinitesimal Euclidean area by a conformal factor and compute the gradient flow for the modified area functional. The derived flow turns out to have two components, one which is constant and one which depends on the conformal factor as well as the evolving curve. When combined with the weighted length minimizing flow derived in $[5,9,10,20]$, we obtain a partial differential equation whose parabolic and hyperbolic components each have consistent interpretations as gradient flows. In application to shape segmentation the new flow exhibits a stronger attraction force to features of interest than the constant inflation term previously used, due to the inclusion of a new doublet term. In fact, in several numerical experiments we used the weighted area flow alone to segment the image. Since this flow requires the computation of only first-order derivatives, it offers significant computational savings over the weighted length minimizing flow.

\section{Weighted Gradient Flows}

The foundation of our approach is the mathematical theory of curves flowing in the plane with speed a function of curvature. In [11, 13, 12], the two key components of this motion were combined into a reactiondiffusion space for shape analysis. More precisely with $\kappa$ the curvature, $\mathcal{N}$ the inward unit normal, and $\mathcal{C}$ the curve coordinates, consider families of plane curves flowing according the equation

$$
\frac{\partial \mathcal{C}}{\partial t}=(\alpha+\beta \kappa) \mathcal{N}
$$

where $\alpha, \beta \in \mathbf{R}, \quad \beta \geq 0$. In this section we shall examine the weighted generalizations of the two parts of this evolution.

\subsection{Curvature Motion}

Referring to Eq. 1, we take $\alpha=0, \beta=1$, and so we get the family of plane curves flowing according to the geometric heat equation

$$
\frac{\partial \mathcal{C}}{\partial t}=\kappa \mathcal{N}
$$

This equation has a number of properties which make it very useful in image processing. In particular, (2) is the Euclidean curve shortening flow, in the sense that the Euclidean perimeter shrinks as quickly as possible when the curve evolves according to $(2)[6,7]$. Since we will need a similar argument for subsequent models, let us work out the details.

Let $\mathcal{C}=\mathcal{C}(p, t)$ be a smooth family of closed curves where $t$ parametrizes the family and $p$ the given curve, say $0 \leq p \leq 1$. (Note we assume that $\mathcal{C}(0, t)=\mathcal{C}(1, t)$ and similarly for the first derivatives.) Define the length functional

$$
L(t)=\int_{0}^{1}\left\|\frac{\partial \mathcal{C}}{\partial p}\right\| d p
$$

Differentiating (taking the "first variation" with respect to $t$ ), and using integration by parts, one can show that

$$
L^{\prime}(t)=-\int_{0}^{L(t)}\left\langle\frac{\partial \mathcal{C}}{\partial t}, \kappa \mathcal{N}\right\rangle d s
$$

where $d s=\left\|\frac{\partial \mathcal{C}}{\partial p}\right\| d p$ denotes arc-length. Thus the direction in which $L(t)$ is decreasing most rapidly is when $\frac{\partial \mathcal{C}}{\partial t}=\kappa \mathcal{N}$. Thus (2) defines a gradient flow.

A much deeper fact is that simple closed curves evolving according to (2) converge to "round" points without developing singularities $[6,7]$.

\subsection{Weighted Length Gradient Flows}

In the recent papers $[5,9,10,20]$, the standard Euclidean metric $d s^{2}=d x^{2}+d y^{2}$ of the underlying space over which the evolution takes place is modified to a conformal metric $d s_{\phi}^{2}=\phi^{2}\left(d x^{2}+d y^{2}\right)$. Using this metric, the " $\phi$-length" of the curve is defined as

$$
L_{\phi}(t)=\int_{0}^{1}\left\|\frac{\partial \mathcal{C}}{\partial p}\right\| \phi d p .
$$

Here $\phi: \mathbf{R}^{2} \rightarrow \mathbf{R}$ is a positive differentiable function defined on the image plane. By requiring the $\phi$-length to shrink as quickly as possible, the following flow is obtained

$$
\mathcal{C}_{t}=\{\phi \kappa-\nabla \phi \cdot \mathcal{N}\} \mathcal{N} .
$$

Note that this last equation consists of two terms. The first is the curvature term of equation (2) multiplied by $\phi$, and the second depends on the gradient of the conformal factor. In application to shape modelling the latter term acts like a doublet which attracts the active contour to the feature of interest. We will now treat the constant speed term of the reaction-diffusion model in the same way. 


\subsection{Constant Motion}

Again, referring to equation (1), we take $\alpha=1, \beta=$ 0 , which gives the constant motion flow

$$
\mathcal{C}_{t}=\mathcal{N}
$$

In analogy to the geometric heat equation which minimizes Euclidean length, this evolution may be derived as the gradient flow which locally minimizes area. Indeed, for the family of closed curves defined above, the area functional is given by

$A(t)=-\frac{1}{2} \int_{0}^{L}\langle\mathcal{C}, \mathcal{N}\rangle d s=-\frac{1}{2} \int_{0}^{1}\left\langle\mathcal{C},\left(\begin{array}{c}-y_{p} \\ x_{p}\end{array}\right)\right\rangle d p$.

Again, taking the first variation

$A^{\prime}(t)=-\frac{1}{2} \int_{0}^{1}\left\langle\mathcal{C}_{t},\left(\begin{array}{c}-y_{p} \\ x_{p}\end{array}\right)\right\rangle d p-\frac{1}{2} \int_{0}^{1}\left\langle\mathcal{C},\left(\begin{array}{c}-y_{p t} \\ x_{p t}\end{array}\right)\right\rangle$

Using integration by parts for the second integral and changing to arc-length parametrization

$$
A^{\prime}(t)=-\int_{0}^{L}\left\langle\mathcal{C}_{t}, \mathcal{N}\right\rangle d s
$$

Thus the direction in which $A(t)$ is decreasing most rapidly (locally) is when $\mathcal{C}_{t}=\mathcal{N}$ and (5) also defines a gradient flow.

Remark. Equations (2) and (5) are guaranteed to give the direction of maximal decrease of their respective functional only locally. Global existence results are much deeper. In fact, we have already stated that a smooth embedded curve shrinking under (2) remains regular, that is, the flow is indeed smoothing. The constant motion flow (5) on the other hand can cause a smooth curve to evolve to a singular one. In fact, this is one of its desirable characteristics since the resulting shocks are important features for the computational theory of shape in $[11,13,12]$.

\subsection{Weighted Area Gradient Flows}

Our strategy, in analogy to what has been done for length, is to consider area in the conformal metric. Hence our starting point is the modified area functional

$A_{\phi}(t)=-\frac{1}{2} \int_{0}^{L(t)} \phi\langle\mathcal{C}, \mathcal{N}\rangle d s=-\frac{1}{2} \int_{0}^{1} \phi\left\langle\mathcal{C},\left(\begin{array}{c}-y_{p} \\ x_{p}\end{array}\right)\right\rangle d p$

Here $\phi: \mathbf{R}^{2} \rightarrow \mathbf{R}$ is a positive differentiable function defined on the image plane. We now derive the flow associated with the $\phi$-area, $A_{\phi}$. As above, differentiating the functional with respect to $t$ will give us the evolution equation for the curve:

$$
\begin{aligned}
-2 A_{\phi}^{\prime}(t) & =\underbrace{\int_{0}^{L}\left\langle\nabla \phi, \mathcal{C}_{t}\right\rangle\langle\mathcal{C}, \mathcal{N}\rangle d s}_{I_{1}}+\underbrace{\int_{0}^{L} \phi\left\langle\mathcal{C}_{t}, \mathcal{N}\right\rangle d s}_{I_{2}} \\
& +\underbrace{\int_{0}^{1} \phi\left\langle\mathcal{C},\left(\begin{array}{c}
-y_{p t} \\
x_{p t}
\end{array}\right)\right\rangle d p}_{I_{3}} .
\end{aligned}
$$

In $I_{1}$ and $I_{2}$ we have changed the parametrization to arc length. For $I_{3}$, using integration by parts, we get

$$
I_{3}=-\int_{0}^{1}\left\langle(\phi \mathcal{C})_{p},\left(\begin{array}{c}
-y_{t} \\
x_{t}
\end{array}\right)\right\rangle d p
$$

We will use the following notation: let $V=(a, b)$ be a $d p$. vector, its "perp" is defined by

$$
V^{\perp}=(-b, a) .
$$

With respect to the scalar product we have the following properties

$$
\begin{aligned}
\left\langle V_{1}, V_{2}^{\perp}\right\rangle & =-\left\langle V_{1}^{\perp}, V_{2}\right\rangle \\
\left\langle V_{1}^{\perp}, V_{2}^{\perp}\right\rangle & =\left\langle V_{1}, V_{2}\right\rangle .
\end{aligned}
$$

Using this, we rewrite $I_{3}$ as follows

$$
I_{3}=-\int_{0}^{1}\left\langle(\phi \mathcal{C})_{p},\left(\mathcal{C}_{t}\right)^{\perp}\right\rangle d p=\int_{0}^{1}\left\langle\mathcal{C}_{t},(\phi \mathcal{C})_{p}^{\perp}\right\rangle d p
$$

But

$$
\begin{aligned}
(\phi \mathcal{C})_{p} & =\left\langle\nabla \phi, \mathcal{C}_{p}\right\rangle \mathcal{C}+\phi \mathcal{C}_{p} \\
\Rightarrow(\phi \mathcal{C})_{p}^{\perp} & =\left\langle\nabla \phi, \mathcal{C}_{p}\right\rangle \mathcal{C}^{\perp}+\phi \mathcal{C}_{p}^{\perp}
\end{aligned}
$$

hence

$$
\begin{aligned}
I_{3} & =\int_{0}^{1}\left\langle\mathcal{C}_{t},\left\langle\nabla \phi, \mathcal{C}_{p}\right\rangle \mathcal{C}^{\perp}+\phi \mathcal{C}_{p}^{\perp}\right\rangle d p \\
& =\int_{0}^{1}\left\langle\nabla \phi, \mathcal{C}_{p}\right\rangle\left\langle\mathcal{C}_{t}, \mathcal{C}^{\perp}\right\rangle d p+\int_{0}^{1} \phi\left\langle\mathcal{C}_{t}, \mathcal{C}_{p}^{\perp}\right\rangle d p .
\end{aligned}
$$

Using equation (6) and changing to arc length parametrization

$$
I_{3}=\int_{0}^{L}\left\langle\mathcal{C}_{t}, \mathcal{C}^{\perp}\right\rangle\left\langle\nabla \phi^{\perp}, \mathcal{N}\right\rangle d s+\int_{0}^{L}\left\langle\mathcal{C}_{t}, \phi \mathcal{N}\right\rangle d s
$$

Grouping everything together, we get

$-2 A_{\phi}^{\prime}(t)=\int_{0}^{L}\left\langle\mathcal{C}_{t},\langle\mathcal{C}, \mathcal{N}\rangle \nabla \phi+2 \phi N+\left\langle\nabla \phi^{\perp}, \mathcal{N}\right\rangle \mathcal{C}^{\perp}\right\rangle d s$ 
Therefore for $A_{\phi}$ to decrease as fast as possible, take

$$
\mathcal{C}_{t}=\phi \mathcal{N}+\frac{1}{2}\left[\langle\mathcal{C}, \mathcal{N}\rangle \nabla \phi+\left\langle\nabla \phi^{\perp}, \mathcal{N}\right\rangle \mathcal{C}^{\perp}\right]
$$

Now decomposing $\nabla \phi$ and $\mathcal{C}^{\perp}$ in the Frenet frame $\{T, \mathcal{N}\}$, and dropping the tangential terms, which can always be done by reparametrizing the curve,

$\mathcal{C}_{t}=\left\{\phi+\frac{1}{2}\left[\langle\nabla \phi, \mathcal{N}\rangle\langle\mathcal{C}, \mathcal{N}\rangle+\left\langle\nabla \phi^{\perp}, \mathcal{N}\right\rangle\left\langle\mathcal{C}^{\perp}, \mathcal{N}\right\rangle\right]\right\} \mathcal{N}$.

The last result can be simplified further. Writing $\nabla \phi=$ $\left(\phi_{x}, \phi_{y}\right), \mathcal{C}(p, t)=(x(p, t), y(p, t))$ and expanding the scalar products we obtain

$$
\langle\nabla \phi, \mathcal{N}\rangle\langle\mathcal{C}, \mathcal{N}\rangle+\left\langle\nabla \phi^{\perp}, \mathcal{N}\right\rangle\left\langle\mathcal{C}^{\perp}, \mathcal{N}\right\rangle=\langle\mathcal{C}, \nabla \phi\rangle
$$

Hence the $\phi$-area minimizing evolution equation takes on the following simple form

$$
\mathcal{C}_{t}=\left\{\phi+\frac{1}{2}\langle\mathcal{C}, \nabla \phi\rangle\right\} \mathcal{N} .
$$

Note that since $\phi$ is a globally defined function on the image plane, (7) defines a hyperbolic equation.

Remark. Whereas in the present work we are modifying the area functional in the usual Euclidean $L^{2}$ sense, it is interesting to note that minimizing the area functional in an affine sense leads to the affine curve shortening flow introduced in $[1,17]$; see [15] for the details. If the area functional is modified by a stopping term, and the minimizing flow is computed again in this affine invariant sense, one gets an affine invariant snake model based on $\kappa^{1 / 3}$, [15]. This is the affine analogue of the work in $[5,9,10,20]$.

\section{Level Set Representations}

In this section we review the level set representation for curves flowing according to functions of curvature, due to Osher and Sethian $[16,18,19]$, which is the basis for their numerical algorithm for curve (and hypersurface) evolution which we utilize. Let $\mathcal{C}(p, t): S^{1} \times[0, \tau) \rightarrow \mathbf{R}^{2}$ be a family of curves satisfying the following evolution equation:

$$
\frac{\partial \mathcal{C}}{\partial t}=S \mathcal{N}
$$

The curve $\mathcal{C}(p, t)$ is represented by the zero level set of a smooth and Lipschitz continuous function $\Psi: \mathbf{R}^{2} \times$ $[0, \tau) \rightarrow \mathbf{R}$, given by $\left\{X \in \mathbf{R}^{2}: \Psi(X, t)=0\right\}$. Since $\mathcal{C}(p, t)$ is on the zero level set, it satisfies

$$
\Psi(\mathcal{C}, t)=0
$$

By differentiating (9) with respect to $t$, and then with respect to the curve parameter $p$, it can be shown that

$$
\Psi_{t}=S\|\nabla \Psi\|
$$

Equation (10) is solved using a combination of straightforward discretization and numerical techniques derived from hyperbolic conservation laws. The curve $\mathcal{C}$, evolving according to (8), is then obtained as the zero level set of $\Psi$. As an example, noting that

$$
\phi+\frac{1}{2}\left(x \phi_{x}+y \phi_{y}\right)=\frac{1}{2} \operatorname{div}\left(\left(\begin{array}{l}
x \\
y
\end{array}\right) \phi\right),
$$

the level set representation of the $\phi$-area minimizing flow (7) is given by

$$
\Psi_{t}=\frac{1}{2} \operatorname{div}\left(\left(\begin{array}{l}
x \\
y
\end{array}\right) \phi\right)\|\nabla \Psi\| .
$$

\section{Shape Modelling}

We now apply a combined length and area minimizing flow to the problem of shape segmentation. We begin with a brief review of earlier work in the area. Caselles et al. [4] and Malladi et al. [14] proposed an active contour model based on the following level set formulation:

$$
\Psi_{t}=\phi(x, y)\left(\operatorname{div}\left(\frac{\nabla \Psi}{\|\nabla \Psi\|}\right)+\nu\right)\|\nabla \Psi\| .
$$

Here the potential $\phi(x, y)$ is constructed to have local minima at edges so that the evolving curve stops at them. For example, in $[4,14] \phi(x, y)$ takes the form:

$$
\phi=\frac{1}{1+\left\|\nabla G_{\sigma} * I\right\|^{n}}
$$

where $I$ is the grey-scale image and $G_{\sigma}$ is a Gaussian smoothing filter. Building upon this work, Tek and Kimia suggested a reaction-diffusion space of bubbles [23] where the key idea is to randomly initialize a number of "seeds," allowing for multiple structures to be captured, such as objects with holes.

It is important to note that in the above methods, the Euclidean curve shortening part of the evolution equation is the gradient flow for shrinking the perimeter of the curve as fast possible; Section 2.1. As explained earlier, in $[5,20,9,10]$ this model is revised and given theoretical justification by replacing the Euclidean metric with a conformal metric one and deriving the associated $\phi$-length gradient flow. In the resulting evolution equation (4) the second term $\langle\nabla \phi, \mathcal{N}\rangle$ acts as a doublet, attracting the curve when it is in the 
vicinity of an edge ${ }^{1}$. Nevertheless, the flow suffers from the practical limitation that the simulation can be extremely slow to converge. As a remedy, the authors of $[5,20,9,10]$ follow $[4,14]$ by adding a constant (hyperbolic) term to keep the curve moving in the desired direction. The calculation of Section 2.4 suggests the modification of replacing the hyperbolic term with the $\phi$-area minimizing flow. The combined equation in level set form is given by:

$$
\begin{aligned}
\Psi_{t} & =\underbrace{\left[\alpha\left(\phi \kappa+\left\langle\nabla \phi, \frac{\nabla \Psi}{\|\nabla \Psi\|}\right\rangle\right)\|\nabla \Psi\|\right]}_{\phi-\text { length }} \\
& +\underbrace{\left[\frac{1}{2} \operatorname{div}\left(\left(\begin{array}{c}
x \\
y
\end{array}\right) \phi\right)\|\nabla \Psi\|\right]}_{\phi \text {-area }} .
\end{aligned}
$$

Note that we have put in a "fudge" factor $\alpha$ in order to make the units compatible (area and length cannot be added) in (15). Since $\phi$ is a globally defined function which depends on the given image, the $\phi$-area part indeed defines a Hamilton-Jacobi equation. Notice by equation (11) that the $\phi$-area minimizing component provides the constant inflationary term (with $\nu=1$ ) used in the earlier approaches to shape modelling $[4,14,23,5,9,10,20]$, as well as a second doublet term which provides an additional attraction force when the front is in the vicinity of an edge. We will illustrate the application of (15) with several numerical simulations of shape segmentation in Section 6 .

\section{Volumetric Extensions}

The 3D extension of our model can be derived as the gradient flow that minimizes an analogous weighted Euclidean volume; see [21] for details. With $\mathcal{S}$ : $[0,1] \times[0,1] \rightarrow \mathbf{R}^{3}$ a compact embedded surface, $\mathcal{N}$ its inward unit normal, and $\phi: \mathbf{R}^{3} \rightarrow \mathbf{R}$ a positive differentiable (stopping) function, the $\phi$-volume minimizing flow turns out to be

$$
\mathcal{S}_{t}=\left\{\phi+\frac{1}{3}\langle\mathcal{S}, \nabla \phi\rangle\right\} \mathcal{N} \text {. }
$$

Note the similarity in form to equation (7).

\section{Remark.}

The equations considered in this paper are special cases of a nonlinear diffusion equation of the form:

$$
\Psi_{t}=\phi(x) a^{i j}(\nabla \Psi) \partial_{i j} \Psi+H(x, \nabla \Psi), \quad x=\left(x_{1}, \ldots, x_{n}\right) .
$$

\footnotetext{
${ }^{1}$ In fact due to the doublet the front will pass back and forth across the edge, but will not stray from it. In contrast in the earlier methods which rely on Eq. 13 the front could eventually pass through and move away from the edge.
}

As in $[2,4,9]$, under mild hypotheses obeyed by our contour models, one may show that

Theorem 1 There is a unique viscosity solution of (16) in $L^{\infty}\left(0, T ; W^{1, \infty}\left(\mathbf{R}^{n}\right)\right)$.

\section{Examples}

An important consideration for the numerical simulation of shape modelling flows is that for any $t$ the image-based stopping term $\phi$ has proper meaning only on the zero level set of the embedding surface $\Psi$. In order to extend this influence to other level sets we use the narrow band technique of [14]. We compute the diffusive $\phi$-length minimizing component using central differences and the hyperbolic $\phi$-area minimizing one using upwind schemes.

As observed in [9] a difficulty with using a large constant motion force $\nu$ (which is desirable for fast convergence) in equation (13) is that it may cause overshooting of the edge since $\phi$ may not be rigorously zero on the contour to be captured. Whereas the stopping behavior of the $\langle\nabla \phi, \nabla \Psi\rangle$ term introduced in $[5,9,10,20]$ can help, in our experience a combined $\phi$-length and $\phi$-area minimizing flow offers at least two advantages. First, the hyperbolic component of the flow is now adaptive, adjusting itself according to the local gradient of the scalar potential $\phi$ in the vicinity of the evolving curve $\mathcal{C}$. Second, the doublet term $\langle\nabla \phi, \mathcal{C}\rangle$ in (15) provides an additional attraction force in the vicinity of edges.

We consider several examples of shape segmentation using a 256x 256 MRI section of a brain, and a $276 \times 268$ CT image of a bone ${ }^{2}$. For all simulations $\phi$ was constructed as in (14), but with curvature based smoothing of the original image [3]. Figure 1 depicts the segmentation of the brain ventricle, with the evolving curve overlayed in white on the curvature smoothed original. We should note that when run with the same parameters $(\Delta t$, and the weights for the parabolic and hyperbolic components), the flow of $[5,9,10,20]$ produces comparable results. The advantages of using the $\phi$-area component emerge when the above flows are applied to the more difficult CT bone image ${ }^{3}$. Whereas our new flow converges on the desired boundary, Figure 2 (left), the flow of $[5,9,10,20]$ eventually "leaks" through, Figure 2 (right). Finally, we use only the $\phi$-area component for segmentation in Figure 3, under three different initial conditions but with the same parameters as in Figure 1. This offers significant computational savings because only first-order derivatives

\footnotetext{
${ }^{2}$ The CT bone image is contrast normalized for display purposes since the original has pixel values ranging only from 0 to 38.

${ }^{3}$ Note that here the direction of the hyperbolic component has been reversed, so it is actually a $\phi$-area maximizing flow.
} 

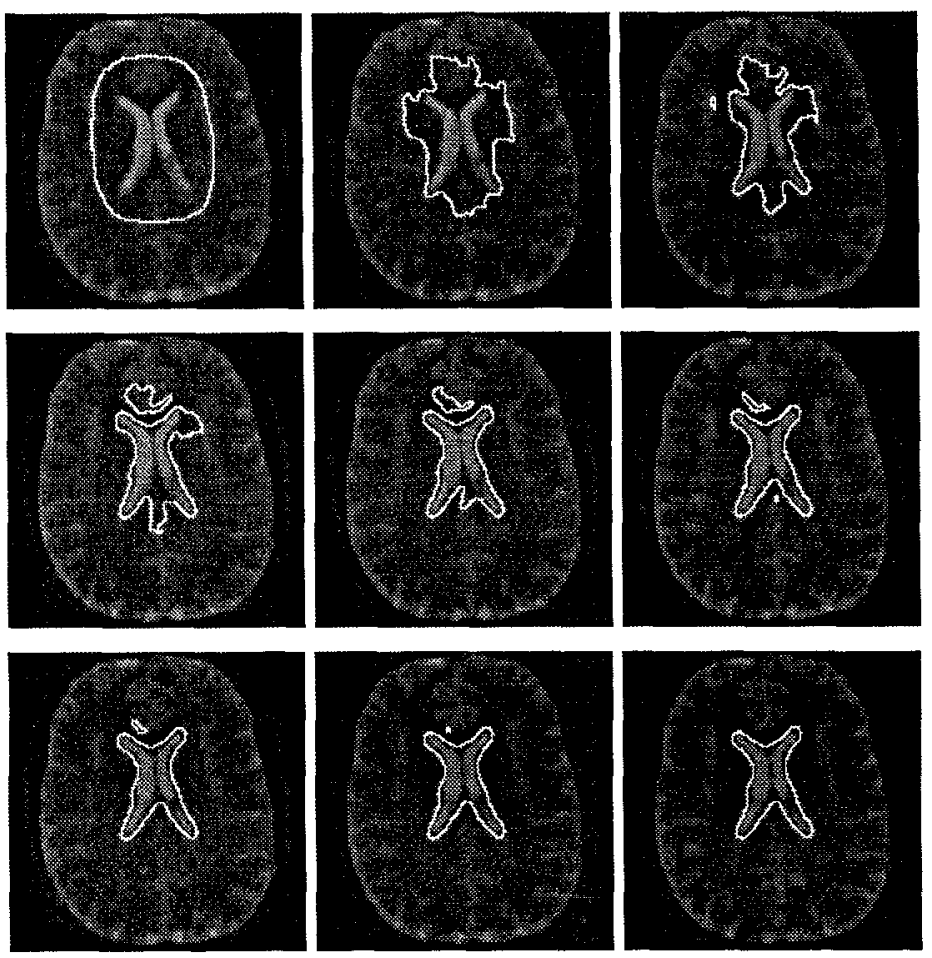

Figure 1: The front evolving under equation (15), overlayed in white, converges on the outline of the brain ventricle. TOP TO BOTTOM, LEFT TO RIGHT: iterations $1,200,400,600,800,1000,1200,1400$, and 1600 .

have to be computed. Also, in most cases the front converges in fewer iterations since it is no longer constrained to remain smooth. We note that although the evolution is not translation invariant due to the doublet term $\langle\nabla \phi, \mathcal{C}\rangle$, the final segmentations in Figures 3 (a) and 3 (b) are basically identical except for the additional piece of noise captured in the latter; observe that in case (a) this structure is not enclosed by the initial contour. The length minimizing component has the advantage of pushing through such "pieces of noise" since they are typically small structures of high curvature.

\section{Conclusions}

Curvature flows have proven to be a powerful tool for a variety of problems in image processing and computer vision. In this paper we have proposed a conformal area based gradient flow for image segmentation and edge finding. This is to be used in conjunction with the conformal length based gradient flows already proposed in the literature $[5,9,10,20]$. As such, we believe that we now have a rather complete picture for the use of such curvature-driven evolution equations for segmentation. Experiments must still be run to study
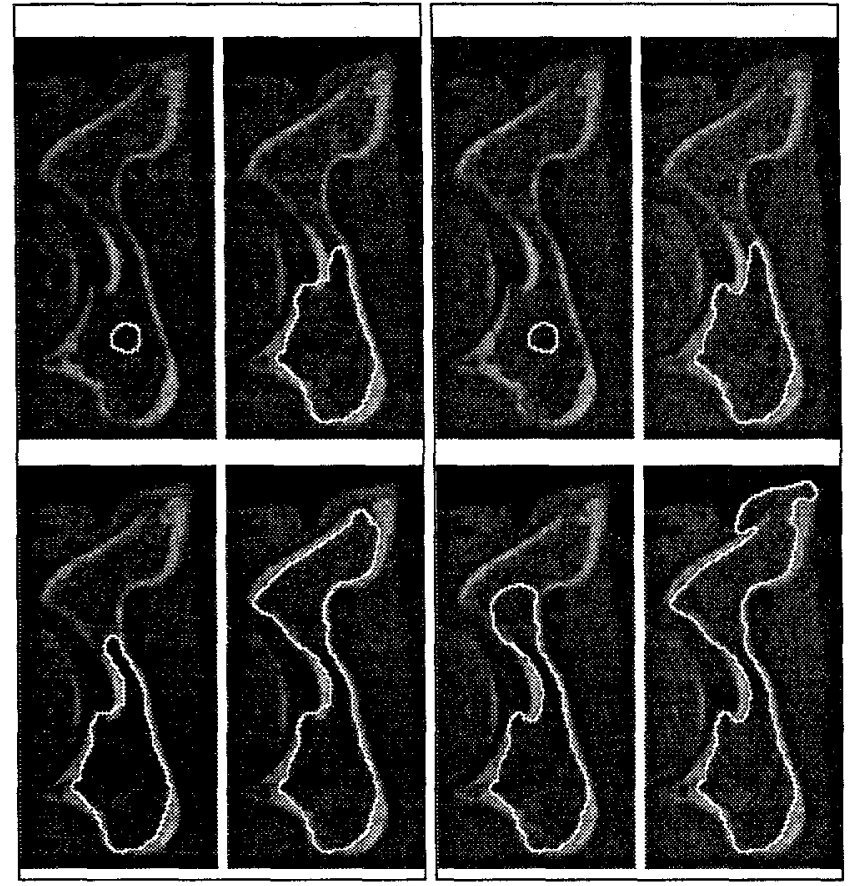

Figure 2: Reversing the direction of the hyperbolic component, the front evolving under equation (15), overlayed in white, converges on the outline of the bone (LEFT). Using identical parameters, the flow of $[5,9,10,20]$ eventually "leaks" through (RIGHT). TOP TO BOTTOM, LEFT TO RIGHT: iterations $1,500,1000$, and 1750 .

the efficacy of the new flow for volumetric imagery.

On the level of shape representation, our flow can be regarded as defining a "conformal skeleton." Indeed, we have been recently employing this flow for a theory of hyperbolic smoothing of shapes, and to define a new "reaction-diffusion" space for planar shape representation. The idea is to use the stopping term to hierarchically remove noise before letting the hyperbolic morphological component take over. This work will be described in our paper [22].

Acknowledgements This work was supported by grants from the Natural Sciences and Engineering Research Council of Canada, from the National Science Foundation, from the Air Force Office of Scientific Research and from the Army Research Office.

\section{References}

[1] L. Alvarez, F. Guichard, P. L. Lions, and J. M. Morel. Axiomatisation et nouveaux opérateurs de la morphologie mathématique. C. R. Acad. Sci. Paris, 315:265-268, 1992. 

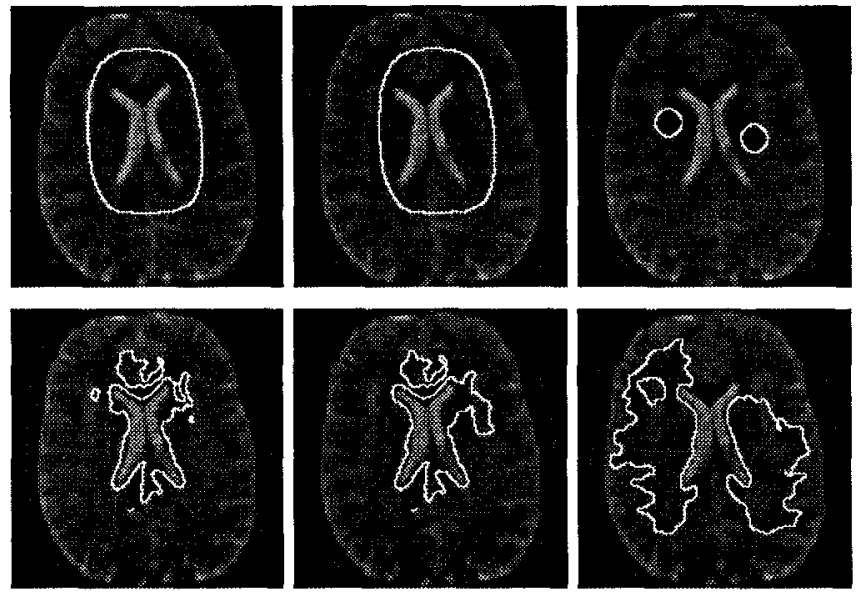

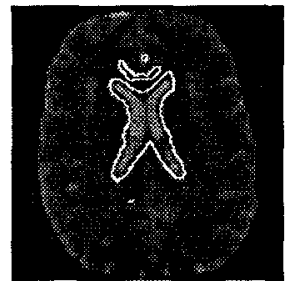

a.

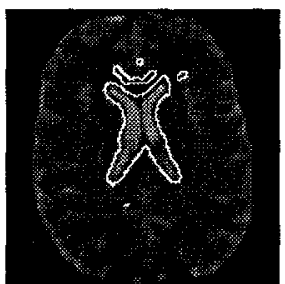

b.

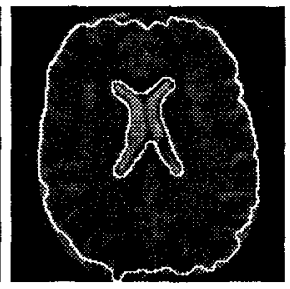

c.
Figure 3: Segmentation of the brain ventricle using only the weighted area term of equation (15). In a) the same initial curve is used as in Figure 1, in b) the initial curve is translated, and in c) an entirely different initial condition is used and the direction of the flow is reversed. TOP то воттом: iterations $1,400,1600$.

[2] - . Axioms and fundamental equations of image processing. Archive of Rational Mechanics and Analysis, 123, 1993.

[3] L. Alvarez, P. L. Lions, and J. M. Morel. Image selective smoothing and edge detection by nonlinear diffusion. SIAM J. Numer. Anal., 29:845-866, 1992.

[4] V. Caselles, F. Catte, T. Coll, and F. Dibos. A geometric model for active contours in image processing. Numerische Mathematik, 66:1-31, 1993.

[5] V. Caselles, R. Kimmel, and G. Sapiro. Geodesic active contours. In Fifth International Conference on Computer Vision, pages 694-699, 1995.

[6] M. Gage and R. Hamilton. The heat equation shrinking convex plane curves. J. Diff. Geom., 23:69-96, 1986.

[7] M. Grayson. The heat equation shrinks embedded plane curves to round points. J. of Differential Geometry, 26:285-314, 1987.

[8] M. Kass, A. Witkin, and D. Terzopoulos. Snakes: Active contour models. $I J C V, 1: 321-331,1987$.

[9] S. Kichenassamy, A. Kumar, P. Olver, A. Tannenbaum, and A. Yezzi. Gradient flows and geometric active contour models. In Fifth International Conference on Computer Vision, pages 810-815, 1995.
[10] -. Conformal curvature flows: From phase transitions to active vision. Archives of Rational Mechanics and Analysis, 134:275-301, 1996.

[11] B. B. Kimia, A. Tannenbaum, and S. W. Zucker. Toward a computational theory of shape: An overview. Lecture Notes in Computer Science, 427:402-407, 1990.

[12] - . On the evolution of curves via a function of curvature I: The classical case. J. of Math. Analysis and Applications, 163:438-458, 1992.

[13] - . Shape, shocks, and deformations I: The components of two-dimensional shape and the reactiondiffusion space. $I J C V, 15: 189-224,1995$.

[14] R. Malladi, J. A. Sethian, and B. C. Vemuri. Shape modeling with front propagation: A level set approach. IEEE TPAMI, 17(2):158-175, February 1995.

[15] P. Olver, G. Sapiro, and A. Tannenbaum. Affine invariant edge maps and active contours. CVIU, submitted.

[16] S. Osher. Riemann solvers, the entropy condition, and difference approximations. SIAM J. Numer. Anal., $21: 217-235,1984$.

[17] G. Sapiro and A. Tannenbaum. On affine plane curve evolution. Journal of Functional Analysis, 119:79-120, 1994.

[18] J. A. Sethian. Curvature and the evolution of fronts. Commun. Math. Phys., 101:487-499, 1985.

[19] - . A review of recent numerical algorithms for hypersurfaces moving with curvature dependent speed. $J$. of Differential Geometry, 31:131-161, 1989.

[20] J. Shah. Recovery of shapes by evolution of zerocrossings. Technical report, Dept. of Mathematics, Northeastern University, Boston, MA, 1995.

[21] K. Siddiqi, Y. B. Lauzière, A. Tannenbaum, and S. W. Zucker. Area and length minimizing flows for shape segmentation. IEEE Transactions on Image Processing, To appear, 1997.

[22] - . Hyperbolic smoothing of shapes. In preparation, 1997.

[23] H. Tek and B. Kimia. Deformable bubbles in the reaction-diffusion space. In Fifth International Conference on Computer Vision, pages 156-162, 1995. 